\title{
Diagnósticos de enfermagem relacionados a agravos cardiovasculares na população em situação de rua de São Paulo
}

RESUMO | OBJETIVO: Elencar diagnósticos de enfermagem que relacionem a falta de conhecimento e as dificuldades de acesso aos serviços de saúde com o desenvolvimento de doenças cardiovasculares. MÉTODO: Estudo exploratório, transversal e quantitativo realizado em São Paulo. Foi aplicado um questionário a 173 voluntários em situação de rua. Os dados foram analisados e associados a Taxonomia de Diagnósticos de Enfermagem Internacional NANDA I, 2018/2020. RESULTADOS: Foi encontrado uma elevação na média de pressão arterial dos estudados. Houve uma grande variação da escolaridade. Foram relatados diversos impedimentos de acesso aos serviços de saúde. Os principais diagnósticos de enfermagem encontrados foram: Conhecimento Deficiente e Saúde deficiente da comunidade. CONCLUSÃO: Com os resultados obtidos, notou-se uma elevação nos valores pressóricos dos voluntários, sendo elencados diagnósticos de enfermagem. Evidenciou-se a necessidade de intervenções em educação, promoção e prevenção a saúde para que, dessa maneira, essa população tenha seus direitos a saúde garantidos.

Palavras-chaves: Doenças cardiovasculares; População em situação de rua; Acesso aos serviços de saúde; Fatores de risco; Fatores socioeconômicos.

\begin{abstract}
OBJECTIVE: To list nursing diagnoses that relate lack of knowledge and difficulties in access to health services to the development of cardiovascular disease. METHODS: Exploratory, cross-sectional and quantitative study conducted in São Paulo. A questionnaire was applied to 173 homeless volunteers. Data were analyzed and associated with NANDA I International Nursing Diagnostic Taxonomy, 2018/2020. RESULTS: An elevation in the mean blood pressure of those studied was found. There was a wide variation in education. Various impediments to accessing health services were reported. The main nursing diagnoses found were Poor Knowledge and Poor Community Health. CONCLUSION: With the results obtained, an elevation in the blood pressure values of the volunteers was noted, and nursing diagnoses were listed. The need for interventions in health education, promotion and prevention was evident, so that this population can have its health rights guaranteed.
\end{abstract}

Keywords: Cardiovascular diseases; Homeless persons; Health services accessibility; Risk factors; Socioeconomic factors.

RESUMEN | OBJETIVO: Enumerar los diagnósticos de enfermería que relacionan el desconocimiento y las dificultades de acceso a los servicios sanitarios con el desarrollo de enfermedades cardiovasculares. MÉTODOS: Estudio exploratorio, transversal y cuantitativo realizado en São Paulo. Se aplicó un cuestionario a 173 voluntarios sin hogar. Los datos fueron analizados y asociados a la Taxonomía Internacional de Diagnóstico de Enfermería NANDA I, 2018/2020. RESULTADOS: Se encontró una elevación de la presión arterial media de los estudiados. Había una gran variación en la educación. Se señalaron varios impedimentos para acceder a los servicios sanitarios. Los principales diagnósticos de enfermería encontrados fueron: Conocimiento deficiente y Salud comunitaria deficiente. CONCLUSIÓN: Con los resultados obtenidos, se observó una elevación de los valores de presión de los voluntarios y se enumeraron los diagnósticos de enfermería. Se evidenció la necesidad de intervenciones en educación, promoción y prevención de la salud, para que esta población tenga garantizados sus derechos sanitarios.

Palabras claves: Enfermedades cardiovasculares; Personas sin hogar; Accesibilidad a los servicios de salud; Factores de riesgo; Factores socioeconómicos.

\section{André Freire Silva}

Bacharel em Enfermagem, Universidade Nove de Julho.

ORCID: 0000-0002-8014-6661

\section{Priscylla Dantas de Almeida Bianco}

Bacharel em Enfermagem, Universidade Nove de Julho.

ORCID: 0000-0003-0879-8099

\section{Andreia Correia de Menezes}

Graduanda em enfermagem, Universidade Nove de Julho.

ORCID: 0000-0002-9407-0418

Recebido em: 08/03/2021

Aprovado em: 28/04/2021
Lais Lorrayne da Silva

Bacharel em Enfermagem Universidade Nove de Julho.

ORCID: 0000-0001-9270-6091

\section{Everaldo Muniz de Oliveira}

MBA em Gestão de Projetos pela Universidade de São Paulo.

ORCID: 0000-0001-8744-666X

\section{Claudia Cristina Soares Muniz}

Coordenadora pedagógica do curso de Enfermagem na Universidade Nove de Julho, Enfermeira pela Universidade Federal de São Paulo, Doutora em Cardiologia pela Universidade de São Paulo. ORCID: 0000-0002-2472-8181
INTRODUÇÃO

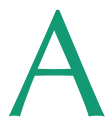
s doenças cardiovasculares (DCV) são comorbidades de causa multifatorial, que acometem o coração e vasos sanguíneos. Esses fatores podem ser divididos entre modificáveis e não modificáveis e entre eles estão: doenças psicossociais, tabagismo, idade, etilismo, obesidade, sedentarismo, hereditariedade, uso de substâncias ilícitas, além de morbidades sem controle como diabetes e hipertensão ${ }^{1,2}$. Essas patologias contemplam as principais causas de morte mundialmente de acordo com 
Organização Pan-Americana da Saúde (OPAS/OMS), sendo o equivalente a $31 \%$ de todas as mortes no mundo em 2015 e a aproximadamente 383.961 mortes no Brasil em 2017 $7^{3,4}$

Segundo o Decreto 7.053 de 23 de Dezembro de 2009, parágrafo único, considera-se população em situação de rua (PSR) o grupo populacional heterogêneo que possui em comum a pobreza extrema, os vínculos familiares interrompidos ou fragilizados e a inexistência de moradia convencional regular, e que utiliza os logradouros públicos e as áreas degradadas como espaço de moradia e de sustento, de forma temporária ou permanente, bem como as unidades de acolhimento para pernoite temporário ou como moradia provisória ${ }^{5}$. Sendo assim, vivenciamos atualmente uma crescente no número dessa massa, sendo que em São Paulo, o último censo de 2019 registrou 24.344 em situação de rua, estes se encontram em maior vulnerabilidade social, tornando-se necessário o levantamento de dados demográficos, estilo de vida, história pregressa e características socioeconômicas detectando a carência de intervenções e a prevalência de Fatores de Risco (FR) de DCV ${ }^{6,7}$.

Desse modo, o processo de enfermagem (PE) é composto por 5 fases, sendo elas: Coleta de dados de enfermagem, Diagnóstico de enfermagem (DE), Planejamento de enfermagem, Implementação e Avaliação de enfermagem. Através dele é possível realizar a assistência de forma sistemática e organizada, garantindo assim um cuidado integral e individualizado. $\mathrm{O}$ DE compreende a $2^{\circ}$ fase do PE e é a interpretação e agrupamento dos dados que foram coletados na primeira etapa somatizados a realização do julgamento clínico sobre as respostas de saúde do indivíduo. Nesse quesito, a Sistematização da Assistência de Enfermagem (SAE), e o DE são essenciais para o julgamento, controle de doenças e conduta terapêutica, identificando intervenções para desenvolver uma eficiente rede de cuidados minimizando riscos e complicações cardiovasculares nessa população ${ }^{8,9,10}$.
A Constituição Federal de 1988 reconhece a saúde como direito de todos e dever do Estado, garantido mediante políticas sociais e econômicas que visem à redução

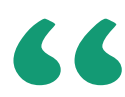

Nesse quesito, a Sistematização da Assistência de Enfermagem (SAE), e o DE são essenciais para 0 julgamento, controle de doenças e conduta terapêutica, identificando intervenções para desenvolver uma eficiente rede de cuidados minimizando riscos e complicações cardiovasculares nessa população do risco de doença e de outros agravos o acesso universal e igualitário às ações e serviços para sua promoção, proteção e recuperação $^{5,11}$. Assim, a legislação brasileira tem a Política Nacional para a População em Situação de Rua de 2009, no qual direciona à atenção à saúde. Dessa maneira, em 2011 por meio dessa instituem-se as equipes de Consultório na Rua, atuando conforme necessidades identificadas nessa população, com ações de promoção, prevenção e educação em saúde ${ }^{12}$.

Segundo o Ministério da Saúde no glossário temático, educação em saúde pode ser definida como "processo educativo de construção de conhecimentos em saúde que visa à apropriação temática pela população e não à profissionalização ou à carreira na saúde, assim como aumentar a autonomia das pessoas no seu cuidado" Sendo assim, fica explicitado a importância desse processo para que ocorra a prevenção de agravos a saúde e aumento da autonomia. Dito isso, foi verificado na PSR os dados relacionados ao acesso nos serviços de saúde, escolaridade e instrução, para que assim, fossem levantados diagnósticos de enfermagem e após isso averiguada a necessidade de ações de enfermagem em promoção e educação em saúde ${ }^{13,14}$.

\section{OBJETIVO}

Elencar diagnósticos de enfermagem que relacionem a falta de conhecimento e as dificuldades de acesso aos serviços de saúde com o desenvolvimento de doenças cardiovasculares.

\section{METODOLOGIA}

Trata-se de uma pesquisa de campo de caráter exploratório, transversal e quantitativo, realizada na Região Central de São Paulo no período de novembro de 2019 a março de 2020. Foi aplicado um questionário previamente estruturado e aprovado pelo Comitê de Ética institucional sob protocolo: 036417, CAAE: 21519413.4.0000.5511. 
Foram 173 voluntários selecionados por conveniência, respeitando a faixa etária de 18 a 60 anos. Para a realização das coletas o horário estabelecido era no

\section{Gráfico 1: Relação do conhecimento dos estudados frente aos fatores de risco para DCV's.}

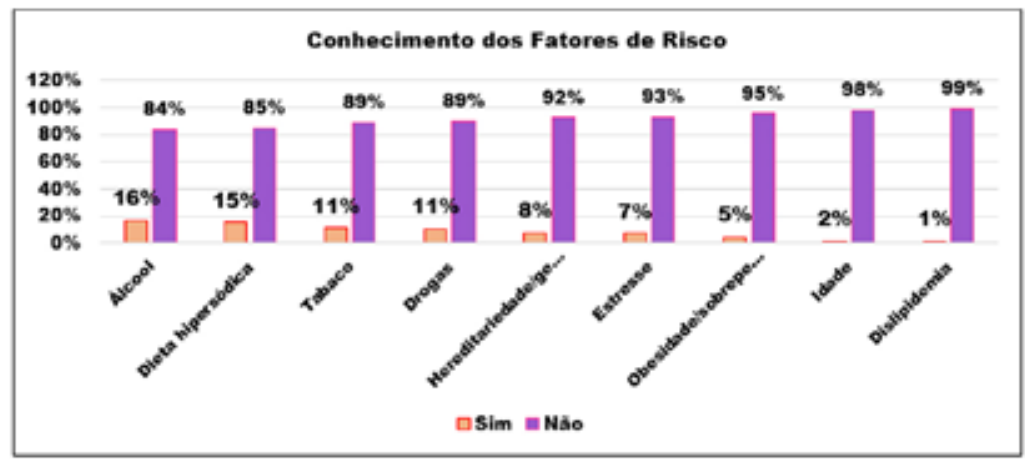

Fonte: dados dos autores

\section{Gráfico 2: Relação da média de PA sistólica, diastólica e FC dos estudados.}

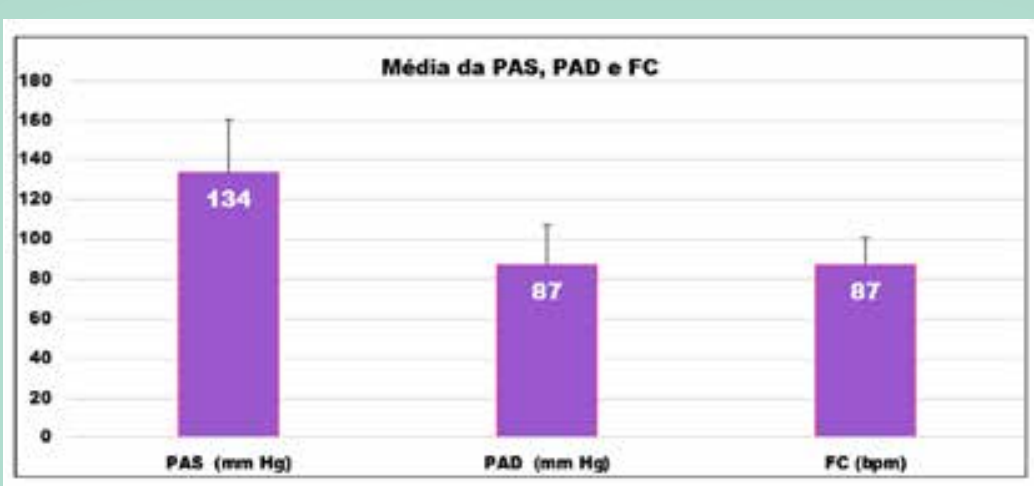

Fonte: dados dos autores

\section{Gráfico 3: Relação da procura de serviços de saúde pelos estudados divididos em gênero.}

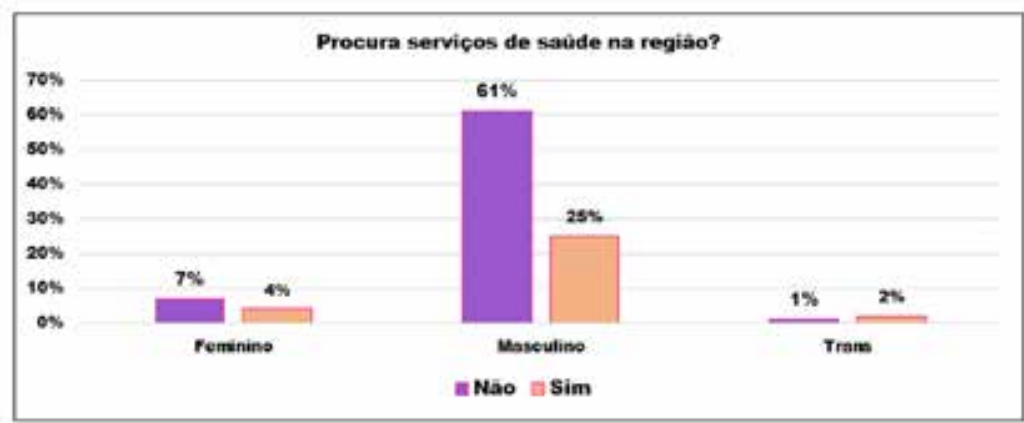

Fonte: dados dos autores sentimento livre e esclarecido, e, caso houvesse o aceite do voluntário, após a assinatura, a entrevista era iniciada.

Eram aplicados os questionários que contêm aproximadamente 50 perguntas relacionadas a saúde cardiovascular, comportamento sexual, autocuidado e dados sociodemográficos, em seguida, realizadas as mensurações da Pressão Arterial (PA) e Frequência Cardíaca (FC), utilizando aparelhos digitais, além das medidas de Circunferência Abdominal (CA), Circunferência Cervical (CC) e altura com fita métrica e do peso com uma balança digital. Os dados foram transferidos para a plataforma digital EXCEL e posteriormente analisados e condensados em forma de tabelas e gráficos.

Esses dados posteriormente foram associados ao livro North American Nursing Diagnoses Association (NANDA) I de 2018-2020, sendo elencados diagnósticos de enfermagem relacionados às dificuldades no acesso aos serviços de saúde, ao conhecimento insuficiente e a agravos cardiovasculares que compreendessem os fatores presentes na população estudada.

\section{RESULTADOS}

Com relação à escolaridade, dos 173 entrevistados, 53\% não estudou sequer até o ensino médio, sendo que destes $53 \%, 5 \%$ afirmaram ser analfabetos, $17 \%$ apenas sabem ler e escrever, 10\% estudou da primeira à quarta série e $21 \%$ do $5^{\circ}$ ao $8^{\circ}$ ano do ensino fundamental. Dos outros $47 \%$ restantes, $16 \%$ não concluíram o $2^{\circ}$ grau do ensino médio e $23 \%$ concluíram, 6\% não concluíram o ensino superior e 3\% têm essa formação.

Os entrevistados foram questionados com relação ao conhecimento prévio de FR para DCV e a maioria não tinha conhecimento dos fatores, tais quais, 99\% para dislipidemia, 98\% idade, 95\% obesidade/sobrepeso, 93\% estresse, $92 \%$ hereditariedade/genética, $90 \%$ drogas, $89 \%$ tabaco, 85\% dieta hipersódica e 84\% álcool. Dados que podem ser observados a 
seguir, no gráfico 1 - Conhecimento dos Fatores de Risco.

Também foram encontradas alterações nos valores de média da PA dos voluntários, sendo a diastólica de $134 \mathrm{mmHg}$, sistólica de $87 \mathrm{mmHg}$ e FC de 87 batimentos por minutos, valores acima do preconizado pela $7^{\circ}$ Diretriz Brasileira de Hipertensão Arterial. O que pode ser verificado no gráfico 2 - Média da PAS, PAD e FC, ao final do parágrafo. Averiguou-se que $72 \%$ informaram nunca ter sido atendido em consulta cardiológica prévia, e, ao observar os que relatam esse atendimento, temos como motivação os diagnósticos de Hipertensão (51\%), Arritmias $(6 \%)$, coronariopatias $(4 \%)$ e insuficiência cardíaca (4\%), sendo apenas $28 \%$ dos estudados.

Notou-se que $69 \%$ dos estudados não frequentam os serviços de saúde, no qual são relatados dificuldades e impedimentos, sendo majoritariamente: Espera no atendimento (33\%), Discriminação (12\%) e Preconceito (10\%), dados evidenciados nos gráficos 3 - Procura serviços de saúde na região? E, no 4 - Algo te impede de frequentar serviços de saúde, logo abaixo. Pontua-se que 91 \% não soube informar a história pregressa de doenças cardiovasculares, evidenciando a pouca compreensão e a dificuldade de acesso aos serviços equitativos a suas necessidades.

Dessa maneira, realizou-se a análise e levantamento dos diagnósticos de
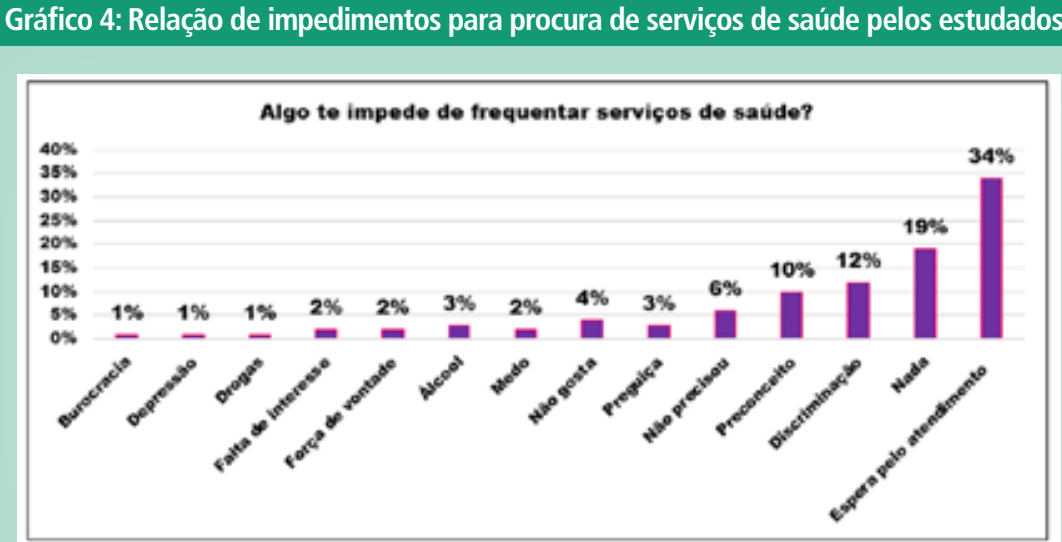

Fonte: dados dos autores enfermagem utilizando a taxonomia I NANDA, relacionando a falta de autocuidado, o risco cardiovascular, as dificuldades no acesso aos serviços de saúde e a baixa compreensão em saúde, encontrando-se:

Saúde deficiente na comunidade caracterizado por problema de saúde vivenciado por grupos ou populações relacionado a acesso insuficiente a provedores de cuidados de saúde.

Conhecimento Deficiente caracterizado por comportamento inadequado, desempenho inadequado em teste, conhecimento inapropriado, relacionado a informações insuficientes.

Comportamento de saúde propenso a risco caracterizado por abuso de substâncias, falha em agir de forma a prevenir problemas de saúde, tabagismo relacionado a apoio social insuficiente e percepção negativa do provedor de cuidados de saúde.

Risco de perfusão tissular cardíaca diminuída relacionado ao risco de abuso de substâncias e conhecimento insuficiente sobre os fatores modificáveis. (North American Nursing Diagnoses, 2018) $)^{15}$.

\section{DISCUSSÃO}

O número de pessoas vivendo em situação de rua em São Paulo é alarmante e seu estado de saúde gera preocupação e inspira cuidados. O conhecimento em saúde é a base para que a prevenção ocorra, evitando assim complicações e internações desnecessárias, diminuindo custos e promovendo saúde. Devido essa fragilidade na compreensão do processo saúde-doença, evidenciou-se a necessidade de intervenções que possam reverter esse quadro, gerando assim promoção e prevenção a saúde que são as bases da Atenção Primária, para quê, com isso, possamos reduzir a morbimortalidade dessa parcela negligenciada da sociedade, sendo capaz de mudar as condições de saúde dessa população.

Com relação ao acesso aos serviços de saúde, constatou-se que a PSR 
tem dificuldades relativas, os levando a procurar principalmente serviços na atenção secundária e terciária, sendo a urgência e emergência e raramente os acompanhamentos relacionados a prevenção e promoção à saúde da atenção primária. Assim, verificou-se que o risco cardiovascular é potencializado, dado que maioria não realiza acompanhamento a saúde, pelas resistências e dificuldades no atendimento, causando a desvalorização social e falta de acesso a serviços de atenção básica. Reconhece-se, que apesar das políticas públicas vigentes, estes possuem menor acesso aos serviços de saúde eficazes e equitativos às suas necessidades, tornando-se necessário a criação, renovação, ampliação e revisão de políticas públicas que assegurem a essa parcela da socie dade a saúde, que de acordo com a lei é direito de todos e dever do Estado.

\section{CONCLUSÃO}

De acordo com o exposto, notou-se que existem diversos fatores que corroboram para os agravos a saúde cardiovascular da PSR, sendo que, dentre eles, estão as dificuldades de acesso aos serviços de saúde e à educação em saúde. Pois, observou-se a falta de conhecimento dos fatores de risco para desenvolvimento de DCV's e, além disso, foram relatados fatores impeditivos ao acesso e a procura de serviços de saúde, estando entre eles o preconceito e a discriminação, o que somatiza na diminuição do autocuidado e controle de comorbidades, elevando assim os riscos para integridade sistêmica.

Percebeu-se que os valores pressóricos desses estudados estavam elevados, evidenciando a influência desses fatores com o surgimento de DCV's, pois sabe-se que a hipertensão arterial é uma doença predisponente a diversas patologias sistêmicas agudas e crônicas, como a insuficiência cardíaca, infartos agudos do miocárdio e acidentes vasculares encefálicos. Dito isso, foram elencados diagnósticos de enfermagem que fundamentaram esses dados, revelando a emergência dos problemas de saúde vivenciados pelos estudados.

E, através disso, nota-se a necessidade explicita de intervenções no âmbito público e político que acarretem na diminuição desses fatores e consequentemente na melhora da qualidade de vida dessa população. Cabendo ao poder público a aplicação das legislações vigentes de maneira a garantir o acesso integral as necessidades de saúde da PSR, demonstrando a importância do fortalecimento, revisão e criação de políticas públicas que contemplem essa parcela negligenciada da sociedade.

\section{Referências}

1. Baggett TP, Liauw SS, Hwang SW. Cardiovascular Disease and Homelessness. journal of the American college of cardiology vol. 71, no. 22, 2018 de the American college of cardiology foundation published de Elsevier [Internet]. [Acesso 2020 Mar 4]; DOI https://doi.org/10.1016/j.jacc.2018.02.077. Disponivel em: https://www.sciencedirect.com/science/article/pii/ S0735109718343237?via\%3Dihub

2. Bourbon M, Natercia M, Astrid MV, Quitéria R. Doenças Cardiovasculares. Repositório Científico Instituto Nacional de Saúde. FEV-2016; Disponível em: http://hdl.handle.net/10400.18/3447

3. Organização Pan-Americana da Saúde (OPAS/OMS), Doenças cardiovasculares, revisado em maio de 2017, [Capturado 9 mar. 2020] disponivelem:https://www.paho.org/bra/index.php?option=com_content\&view=article\&id=5253:doencas-cardiovasculares\&ltemid=1096 4. Sociedade Brasileira de Cardiologia (SBV), Cardiômetro, [Capturado: 10 Dez. 2020]. Disponível em: http://www.cardiometro.com.br/anteriores.asp. 5. DECRETO No 7.053 DE 23 DE DEZEMBRO DE 2009. [Capturado: 10 Dez. 2020]. Disponivel em: http://www.planalto.gov.br/ccivil_03/_Ato20072010/2009/Decreto/D7053.htm

6. Prefeitura de São Paulo divulga Censo da População em Situação de Rua 2019, Secretaria Especial de Comunicação (31/01/2020). [Capturado 9 mar. 2020] Disponível em: http://www.capital.sp.gov.br/noticia/prefeitura-de-sao-paulo-divulga-censo-da-populacao-em-situacao-de-rua-2019

7. Hino P, Santos JO, Rosa AS. Pessoas que vivenciam situação de rua sob o olhar da saúde. Rev Bras Enferm, São Paulo, v. 71, n. 1, p. 684-92, Ago 2018. DOI: http://dx.doi.org/10.1590/0034-7167-2017-0547. Disponivel em: https:// www.scielo.br/pdf/reben/v71s1/pt_0034-7167-reben-71-s1-0684.pdf. Acesso em: 7 mar. 2020.

8. Conselho Federal de Enfermagem (COFEN). RESOLUÇÃO COFEN-358/2009. [publicação na we]. Out.2009 acesso em 03 de março de 2020. Disponível em: http://www.cofen.gov.br/resoluo-cofen-3582009_4384.html
9. Pinto ESO, Rodrigues WN, Sistematização da Assistência de Enfermagem na Atenção Primária a Pessoas Portadoras de Hipertensão Arterial. Rev. Nursing 2018,21(237): 2036-2040. Disponivel em: http://www.revistanursing.com.br/revistas/237Fevereiro2018/sistematizacao_da_assistencia_de_ enfermagem.pdf

10. BRAGA, J. L. Prevalência dos diagnósticos de enfermagem manifestados por pessoas com problemas cardiovasculares: uma revisão integrativa. 2019. 62 f. Monografia (Graduação em Enfermagem) - Faculdade de Farmácia, Odontologia e Enfermagem, Universidade Federal do Ceará, Fortaleza, 2019. Disponivel em: http://repositorio.ufc.br/handle/riufc/43589

11. Legislação, Constituição Federal (Texto compilado até a Emenda Constitucional $n^{\circ} 101$ de 03/07/2019), Art. 196. [Capturado: 10 Dez. 2020] Disponivel em: https://www.senado.leg.br/atividade/const/con1988/ con1988_03.07.2019/art_196_.asp

12. Ministério da saúde, Secretaria de Atenção Primaria a saúde, Consultório na rua, [Capturado: 10 Dez. 2020]. Disponível em: https:// aps.saude.gov.br/ape/consultoriorua/\#: :text=A\%20estrat $\%$ C3\%A9gia $\% 20$ Consult $\%$ C3\%B3rio $\% 20$ na $\% 20$ Rua, encontra $\% 20$ em $\% 20$ condi $\%$ C3\%A7\%C3\%B5es\%20de\%20vulnerabilidade

13. Ministério da Saúde, Glossário Temático: Gestão do Trabalho e da Educação na Saúde, $2^{\text {a }}$ edição, Brasilia - DF, 2013, pág. 19. Disponível em: http:// bvsms.saude.gov.br/bvs/publicacoes/glossario_tematico_gestao_trabalho_ educacao saude_2ed.pdf

14. Chaves Junior P.R.; Aguiar R.S. Compreensão e entendimento de saúde vivenciado por pessoas em situação de rua. Revista Nursing, São Paulo, v $23 \mathrm{n}$ 263 p. 3688-3692, abr. 2020. ID: biblio-1100508 Disponivel em: https://pesquisa.bvsalud.org/portal/resource/pt/biblio-1100508. Acesso em: 7 jan. 2020. 15. Herdman T. H.; Kamitsuru S. Diagnósticos de Enfermagem da NANDA I Definições e Classificação 2018-2020. Tradução: Regina M. Garcez. 11. ed. Porto Alegre: Artmed, 2018. 149, 255, 143, 230 p. 